\title{
MedienPädagogik
}

www. medienpaed.com

Zeitschrift für

Theorie und Praxis

der Medienbildung

ISSN 1424-3636

\section{Zur didaktischen Konzeption von «Sozialen Lernplattformen» für das Lernen in Gemeinschaften}

\author{
Michael Kerres, Tobias Hölterhof und Axel Nattland
}

\begin{abstract}
Mit der aktuellen Diskussion über Web 2.0 stellt sich die Frage nach der Zukunft von traditionellen Lernplattformen. Der Beitrag stellt verschiedene Entwicklungsoptionen vor und beschreibt die Perspektiven «sozialer Lernplattformen». Bei diesen stehen die Aktivitäten der Akteure und ihre Interaktion im Vordergrund und nicht Dokumente und Lernmaterialien. Sie sind durchlässig für Informationen aus dem und in das Internet, woraus sich unterschiedliche Optionen für die Privatheit von Informationen ergeben. Schliesslich unterstützen sie die Zusammenarbeit, die Entwicklung von Bekanntschaften und den Aufbau sozialer Gruppen. Dabei stellt sich die Frage, wie diese sozialen Strukturen auf einer Plattform zu konzipieren sind, um Gemeinschaftsbildung beim Lernen zu fördern.
\end{abstract}

\section{$1 \quad$ Einleitung}

Traditionell werden Lernplattformen ${ }^{1}$ gesehen als Orte im Internet, die Ressourcen für das Lehren und Lernen bereitstellen: Neben Lernmaterialien beinhalten sie Werkzeuge für die Kommunikation und das gemeinschaftliche Erstellen von Artefakten. Die häufigste Aktivität auf Lernplattformen, wie Blackboard oder Moodle, ist dabei in der Regel das Einstellen und Verteilen und von Dokumenten und Lernressourcen (vgl. Petschenka und Engert 2011). Seltener gelingt es, eine soziale Lernsituation auf der Plattform zu gestalten, in denen Lernende gemeinschaftlich an Aufgaben arbeiten, sich austauschen und in Gruppen beschäftigt sind. Mit den stark wachsenden sozialen Plattformen und Web-2.0-Anwendungen, die sich jenseits der Lernplattformen entwickeln, stellt sich die Frage nach der Zukunft traditioneller Lernplattformen (vgl. Kerres 2006).

2009 fand beispielsweise ein Workshop in Grossbritannien auf der ALT-C-Tagung statt mit der provozierenden Frage: «The VLE is dead?!» ${ }^{2}$, in der die Zukunft von Lernplattformen kritisch diskutiert wurde: Lernende und auch Lehrende greifen in ihrem Personal Learning Environment zunehmend auf Web-2.0-Werkzeuge zu, um mit Materialien und Dokumenten - auch mit Anderen - zu arbeiten und diese dort

1 engl. Learning Management System (LMS)

2 VLE = virtual learning environment; http://altc2010.alt.ac.uk/ s.a. die Diskussion zur «Post-LMS» Ära: http://www.educause.edu/EDUCAUSE+Quarterly/EDUCAUSEQuarterlyMagazineVolum/EnvisioningthePostLMSEraTheOpe/199389 
verfügbar zu machen (s. a. Conole und Alevizou 2010). Viele der Grundfunktionen, wie sie LMS bieten, sind über offene Plattformen - zumeist ohne Kosten für die Nutzenden - frei verfügbar: Für das Einstellen und Verteilen von Dokumenten (seien es Bilder, Videos, Skripte, Texte, Mindmaps, Audiobeiträge etc.) stehen diverse Plattformen zur Verfügung, deren Inhalte sich z. B. über Schnittstellen und Feeds auslesen und in der persönlichen Arbeitsumgebung zusammenführen lassen. Gerade für die Zusammenarbeit in Gruppen, kollaboratives Lernen, synchrone Kommunikation und Online-Konferenzen existieren leistungsfähiger Werkzeuge, mit denen Lernende zunehmend gewohnt sind zu arbeiten.

Es stellt sich die Frage, wie Bildungsanbieter und Institutionen mit dieser Entwicklung umgehen sollen? Hochschulen etwa betreiben in der Regel eine komplexe IT-Infrastruktur für «Studium und Lehre». Diese umfasst deutlich mehr als ein LMS. Neben den technischen Basisdiensten wie E-Mail, Dokumenten-oder Archivierungs-Server gehören hierzu u. a. die Software-Anwendungen für die Studierenden- und Prüfungsverwaltung, die Zugänge zu Ressourcen der Bibliothek, Lösungen für die Durchführung von PC-gestützten Prüfungen etc. etc. (vgl. Kerres et al. 2009). Dazu existiert die unüberschaubare Vielfalt an Online-Werkzeugen, die einige der Funktionen für das Lehren und Lernen technisch durchaus übernehmen können und die Lehrende und Lernende in ihrem Alltag auch zunehmend nutzen. Die Arbeitsumgebung des Lernenden und Lehrenden ist immer weniger das LMS des Bildungsanbieters. Das LMS wäre präziser als die Lehrplattform des Bildungsanbieters zu bezeichnen, die Lernangebote bereithält, die die Lernenden letztlich in ihrer persönlichen Lernumgebung mit verschiedenartigen Werkzeugen - auch mit Anderen - bearbeiten.

Soll man in dieser Situation als Bildungsanbieter überhaupt ein LMS vorhalten oder konsequent auf Web-2.0-Plattformen setzen? Weiter gedacht geht es auch um die Frage, ob mit dieser Entwicklung nicht auch eine grundlegende Verschiebung einhergeht, weg von formellen Bildungsanbietern hin zu offenen Bildungsangeboten, die sich auf sozialen Plattformen im Netz etablieren?

\section{Was ist «Soziales Lernen»?}

Die aktuelle Diskussion um social learning ist eng verknüpft mit der Entwicklung von sozialen Netzwerken im Internet (wie Linkedln, XING, Facebook) und SocialMedia-Plattformen (wie YouTube, Slideshare, Scribd), bei denen Menschen sich mit anderen austauschen und Informationen teilen. Es entstehen grosse Mengen an Ressourcen, die für das Lernen genutzt werden, zum Beispiel im Rahmen von Lehrgängen und anderen institutionellen Bildungsangeboten. Besonders interessant sind diese Ressourcen auch für solche Lernprozesse, die nicht an eine lehrende Institutionen gebunden stattfinden: autodidaktisch, selbstgesteuert, in sozialen Netzwerken und im Austausch von Wissen «peer-to-peer». Manche dieser Aktivitä- 
ten würden die Beteiligten gar nicht als «Lernen» etikettieren, wenn es nicht-intentional oder implizit geschieht, wie z. B. in der Zusammenarbeit und im Austausch mit anderen, in der Projektarbeit und in informellen Kontakten.

Siemens (2009) betont die Bedeutung des sozialen Austauschs für die Fähigkeit von Unternehmen sich an wachsende Umweltbedingungen anzupassen. ${ }^{3}$ Doch auch in der Diskussion über lebenslanges Lernen wird die Bedeutung solcher Formen von Lernaktivitäten für Individuen, Organisationen und die Gesellschaft zunehmend erkannt. Lebenslanges Lernen bezieht sich nur zu einem vergleichsweise geringen Teil auf z. B. die Teilnahme an Weiterbildungskursen, sondern schliesst auch die aktive Partizipation an kulturellen Wissensressourcen und gesellschaftlicher Wissenskommunikation ein. Digitale Medien und das Internet spielen dabei eine zunehmend wichtige Rolle, für den Zugang zu kulturellen Wissensressourcen und zur Teilhabe an gesellschaftlicher Wissenskommunikation. Hiermit erweitert sich die Diskussion in der Weiterbildungsforschung, die traditionell eher institutionalisierte Formen von Allgemeiner Erwachsenenbildung und beruflicher / betrieblicher Weiterbildung fokussiert (vgl. Nuissl 2010; Prenzel et al. 1997; Tippelt 2010). Dabei ist zu beachten, dass die Begriffe des «sozialen Lernens» und einer «sozialen Lerntheorie» sehr unterschiedlich verwendet werden können und der gemeinte Bezug stets genau zu benennen ist. In der psychologischen Literatur werden mit dem Begriff der sozialen Lerntheorie etwa insbesondere die Arbeiten von Bandura verbunden (Bandura 1971, 1978; s. a. Grusec 1992). Bandura hatte aufgezeigt, dass Lernen nicht nur als Folge von Reaktionen der Umwelt stattfindet, sondern auch durch Beobachtung des Verhaltens Anderer. Damit schaffte er die Grundlage für eine sozial-kognitive Erklärung des Lernens, die sich von einer traditionellen behavioristischen Sicht abhob.

In der Diskussion über Didaktik kann sich «soziales Lernen» dagegen auf folgende ganz unterschiedliche Sachverhalte beziehen:

- auf einen bestimmten Lerngegenstand und Lehrziele, die mit dem Erlernen von Sozialverhalten zusammenhängen (Rollenübernahme, Gesprächsführung, Empathie, Respekt, Verantwortung, Teamfähigkeit etc.),

- auf eine bestimmte didaktische Methode: z.B. das kooperative Lernen, bei dem gemeinsam an einem fachlichen Thema gearbeitet wird,

- auf verschiedene technische Medien und Werkzeuge, die den kommunikativen Austausch und das Zusammenarbeiten von Lernenden unterstützen (vgl. Haake et al. 2011): z. B. Wikis oder Videokonferenzen, oder

- auf eine bestimmte Lernorganisation und das Lernen in einem sozialen Kontext / mit anderen Menschen («Peers»).

3 http://internettimealliance.com/white_papers/Social\%20Learning\%20ITA-jc.pdf 
In der deutschsprachigen Diskussion, wie etwa bei Tillmann (1976; s. a. Baacke 1985; Oelkers 1982; Petillon 1993), wird «soziales Lernen» vor allem auf Lehrziele im Zusammenhang mit der Entwicklung von Sozialkompetenz bezogen. Zugleich wird der Begriff des sozialen Lernens teilweise auch als Synonym für solche didaktische Methoden benutzt, die sich auf gemeinschaftliche Lernaktivitäten in Gruppen beziehen. Kooperatives Lernen ist beispielsweise eine didaktische Methode, bei der die Zusammenarbeit der Lernenden eine eher instrumentelle Funktion hat, um ein bestimmtes fachliches Lehrziel (jenseits des Sozialverhaltens) zu erlernen, z. B. eine Fremdsprache.

Schliesslich kann «soziales Lernen», wie erwähnt, als Variante der Lernorganisation begriffen werden und meint dann die Einbettung von Lernen in einen sozialen, ggfs. auch institutionellen Kontext und mit anderen Lernenden («Peers»). Das «soziale Lernen» ist dabei abzugrenzen auf der einen Seite von einem «autodidaktischen Lernen», bei dem sich die Lernenden einen Lerngegenstand selbständig ohne eine Einbettung in einen weiteren sozialen und / oder institutionellen Kontext aneignen. Auf der anderen Seite steht das «betreute Lernen», bei dem eine Lehrperson oder lehrende Institution den Lernprozess unterstützt.

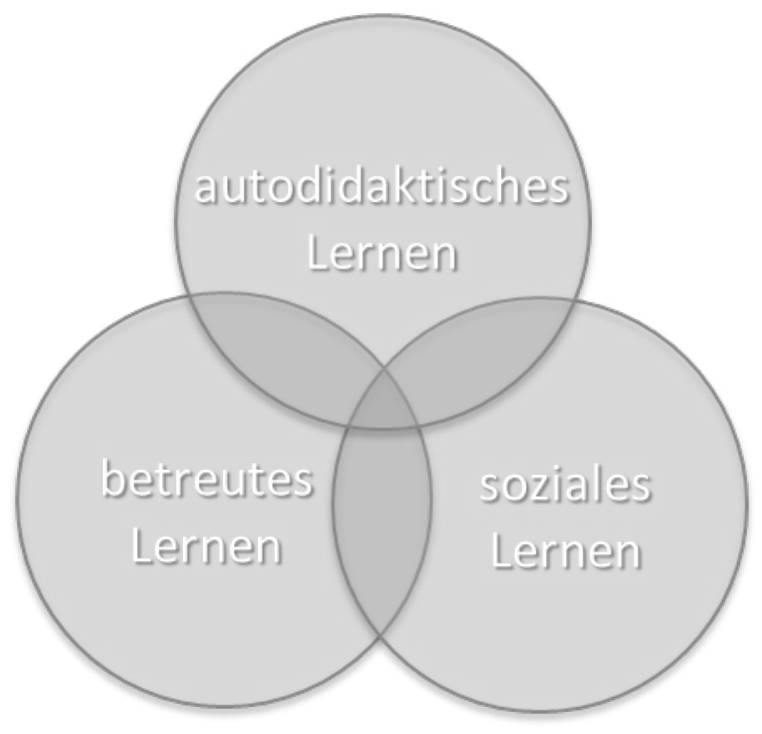

Abbildung 1: Varianten der Lernorganisation

Abbildung 1 zeigt, dass die Übergänge zwischen den einzelnen Bereichen fliessend sind: «Soziales Lernen» meint im Folgenden ein Variante der Lernorganisation, bei der das Lernen in einem sozialen Kontext im Austausch «mit Anderen» stattfindet, etwa im Seminarraum, aber ebenso am Rande einer Tagung mit Kollegen, in einer Projektgruppe in einem betrieblichen Arbeitskontext oder in einer 
Lerngemeinschaft im Internet. Das Soziale Lernen kann dabei von einer lehrenden Instanz betreut werden oder weist eher autodidaktische Züge, wenn keine lehrende Instanz den Lernprozess unterstützt. ${ }^{4}$

Soziales Lernen wird im Folgenden als Variante der Lernorganisation diskutiert und ist damit nicht identisch ist mit einer didaktischen Methode oder gar bestimmten technischen Medien. Es kann zum Beispiel ein sozialer Lernkontext vorliegen, in der eine Gruppe von Lernenden sich persönlich kennt und sich in unterschiedlichen Formen, z. B. ausserhalb des Unterrichts, austauscht. Und dennoch kann das Lernangebot in diesem Rahmen der Methode des Vortrags folgen und keine, zum Beispiel, Gruppenarbeit einsetzt.

Soziales Lernen kann dabei innerhalb formeller Bildungsangeboten einer Institution stattfinden aber ebenso auch jenseits von Kursen, Lehrgängen oder Veranstaltungen als informelles Lernen. Lange Zeit war das soziale Lernen eng verknüpft mit institutionellen Bildungsangeboten von Schule, Hochschule oder Einrichtungen der Erwachsenenbildung: Man musste einen Kurs aufsuchen, um mit anderen zu lernen und um ein soziales Umfeld zu finden, das die eigenen Lernaktivitäten stützt. Das Besondere an der aktuellen Entwicklung ist, dass sich soziales Lernen durch Communitys und das Internet, durch Wissens- und Kommunikationsplattformen und soziale Netzwerke davon zum Teil löst, und Menschen zunehmend alternative Wege des Lernens finden - zum Teil ohne jegliche institutionellen Formen der Strukturierung und Betreuung.

Zusammengefasst lassen sich folgende Gründe nennen, die für soziales Lernen als Variante der Lernorganisation sprechen können:

1. Bestimmte Lehrziele lassen sich nur in sozialen Settings erreichen: z. B. Fertigkeiten in der Gesprächsführung, Teamfähigkeit etc. Andere Lernende können dabei Übungspartner/innen oder auch Rollen-Vorbilder sein, um Sozialverhalten zu erlernen.

2. Bestimmte didaktische Methoden erfordern ein Partner- oder Gruppenkonstellation: z. B. das kooperative Lernen mit Lernaufgaben. So sind Lernerfahrungen möglich, die in einer autodidaktischen Situation nicht realisierbar wären.

3. In Gruppen entstehen Beziehungen zwischen Menschen, die den Lernort und das Lernen selbst aufwerten und interessanter machen. Es zeigt sich, dass dies die Gefahr des Abbruchs von Lernaktivitäten («Dropout») senken kann.

4. Andere Lerner sind Wissensressourcen, die zusätzliche (authentische) Sichten und unterschiedliche Fertigkeiten für die Bearbeitung von Gruppenaufgaben einbringen, die sie in anderen Kontexten erworben haben.

4 Im Kontext der sozio-kulturellen Theorie des Lernen könnte jedes Lernen als «sozial» charakterisiert werden: Auch beim Lernen etwa mit einem Buch partizipiert der Einzelne an kulturell eingebettetem und letztlich über Andere vermitteltem Wissen. Die folgende Diskussion beschränkt sich jedoch auf Lernsituationen, in denen Andere («Peers») in der Lernsituation präsent sind (vgl. Salomon und Perkins 1998). 
5. Durch den Austausch von Menschen in Gruppen entstehen Impulse für das Lernen von Organisationen. Dies trägt zur Entwicklung von Organisationen bei, auch jenseits des Lernens des Einzelnen.

6. Durch die Teilhabe an Wissensgemeinschaften vernetzen sich Lernende mit Anderen. Es öffnet sich der Zugang zu kulturellem Wissen und gesellschaftlicher Kommunikation.

Alle diese Argumente für soziales Lernen können die Nutzung von sozialen Lernplattformen im Internet begründen. Allerdings wird man im konkreten Fall nicht alle Argumente heranziehen, sondern sollte eine Begründung aus Lehrzielen und der gewählten didaktischen Methode ableiten. Insbesondere der erste Grund, der sich auf die Entwicklung von Sozialverhalten bezieht, wird allerdings in der Regel eine face-to-face Situation erfordern.

In dieser Auflistung wird deutlich, dass in diesen Überlegungen ganz unterschiedliche theoretische Argumentationsstränge zusammenkommen. Salomon und Perkins (1998) beschreiben auf der einen Seite Ansätze, die den Anderen letztlich als «facilitator» oder «mediator» für den Wissenserwerb des Einzelnen sehen. Auf der anderen Seite stehen Ansätze, die Lernen immer als einen sozial-konstruktiven Vorgang sehen, bei dem der Einzelne an kulturell eingebettetem Wissen partizipiert. Zunehmend finden sich Verweise auf Ansätze, in denen sozio- konstruktivistische und auch post-humanistische Sichten im Mittelpunkt stehen. Der individuelle Wissenserwerb tritt zurück gegenüber der Re-Konstruktion durch Teilhabe und der Stärkung des Wissensnetzwerkes selbst. Da immer mehr Wissen im Netz verteilt vorliegt (vgl. zu «distributed cognition» Salomon 1993), wächst die Bedeutung der Fertigkeiten der Wissenserschliessung und des Vernetzseins gegenüber der aktuellen Verfügbarkeit von Wissen für das Individuum. Wenn alles Wissen für Alle im Netz verfügbar sein soll, muss das Netz und die kollektive Intelligenz gestärkt werden und nicht mehr (nur) das Individuum (Yang 2010). Das Individuum muss zugleich dann vor allem über genau jene Fertigkeiten verfügen, sich als Teil des Netzes bewegen und Informationen re-konstruieren zu können, um Wissen zu finden und zu bewerten und sich selbst in angemessener Weise als Wissensressource einbringen zu können (vgl. Downes 2010).

Siemens (2009) bezeichnet Lernen als eine Aktivität der Netzwerkbildung, bei der verfügbare Wissensbestände aufgegriffen und durch neue Verknüpfungen im Netzwerk rekonstruiert werden. Dies geschieht im Prinzip auch im traditionellen Unterricht: Der Lernende nimmt Wissen auf und rekonstruiert es in seinen Aktivitäten. Online-gestützte Verfahren der Wissens(re)konstruktion stellen solches Wissen aber immer wieder dem Netz und damit Anderen (oder Allen) zur Verfügung. 


\section{Perspektiven für künftige Lernplattformen}

Es wurde beschreiben, dass Lernplattformen durch die Diskussion über soziale Plattformen und die Forderungen nach sozialem Lernen herausgefordert sind. Es lassen sich drei mögliche Routen erkennen, um perspektivisch mit dieser Herausforderung umzugehen:

\section{a) Verzicht auf Lernplattform}

Die konsequente Nutzung von Web-2.0-Werkzeugen kann mit einem vollständigen Verzicht auf eine Lernplattform einhergehen. Es sind hinreichend Beispiele bekannt, in denen E-Learning Angebote erfolgreich realisiert werden ohne Nutzung eines LMS (vgl. Brown 2010).

Die Lehrperson kann dabei etwa folgendermassen vorgehen (s. a. Conole und Alevizou 2010):

- Eigene Dokumente und Materialien werden auf eine Plattform, wie Scribd oder Slideshare, eingestellt.

- Verfügbare Materialien werden auf Plattformen wie YouTube oder Flickr gesucht.

- Verweise auf alle relevanten Materialien werden mit einem BookmarkingDienst, wie z. B. Diigo, gespeichert.

- Den Lernenden wird die Bookmark-Liste per E-Mail zugesendet. Sie bilden zusammen mit der Lehrperson eine Gruppe im Bookmarking-Dienst, um weitere Materialien und Fundstücke zu teilen und gemeinsam zu verwalten.

- Alternativ dazu kann die Lehrperson eine eigene Webseite für den Kurs einrichten, zum Beispiel über einen kostenlosen Blog-Dienst, und die Verweise dort einstellen.

- Oder die Lehrperson lädt zu einem Wiki (wie z. B. Etherpad) ein, in der sie die Verweise einträgt und die Lernenden auffordert, weitere Materialien und Fundstücke dort einzutragen.

- Schliesslich könnte sie (Teile) dieser Dienste auch zusammenführen auf einer Anwendungs-Suite im Internet, wie Google Apps (für Education), Microsoft Live oder Zoho Apps.

Ein solches Szenario ist gut zu realisieren bei informellen Lernaktivitäten - jenseits von institutionellen Bildungsangeboten, zum Beispiel wenn sich Menschen treffen und im Netz an einem Thema gemeinsam lernen und arbeiten wollen. Darüber hinaus ist dieses Szenario insbesondere auch dort möglich, wo eine engere persönliche Beziehung zu den Lernenden besteht, wie z. B. in der Schule oder in der Erwachsenenbildung. Das eigentliche Management des Kursablaufes, die zuverlässige Vereinbarung von Terminen, Prüfungen und die Notenvergabe, die per- 
sönliche Kommunikation - auch bei Problemen - kann face-to-face erfolgen. Das Arbeiten mit Web-2.0-Werkzeugen erfolgt parallel dazu und erfordert nicht zwingend einen Ort im Internet, der als zentrale Anlaufstelle für die Organisation der Lehr-Lernaktivitäten dient. Ein komplettes Fernstudium dagegen wird man nicht gerne in dieser Weise organisieren wollen.

\section{b) Community-Werkzeuge}

Alternativ können Lernangebote, die stark auf eine sozial-kommunikative Komponente setzen, primär auf einer Community-Plattform statt auf einer Lernplattform realisiert werden. Es existiert eine Fülle an Anbietern, die - zumindest für den Einstieg - eine für die Nutzenden kostenfreie Umgebung bieten. ${ }^{5}$ Auf diesen Community-Plattformen sind verschiedene Funktionen für soziale Interaktion und Kommunikation implementiert: Man stellt Informationen über sich ein und kann Informationen über Andere einsehen. Es lassen sich Beiträge und Materialien einstellen, kommentieren und oft auch bewerten. Andere Personen können als «friends» gekennzeichnet werden und man kann Gruppen bilden, in denen man sich mit Anderen austauscht.

Community-Plattformen bieten darüber hinaus zum Teil ausgesprochen ausgeklügelte Mechanismen des Reputationsmanagements, mit denen Teilnehmende motiviert werden sollen, sich aktiv in der Community zu beteiligen (vgl. Peters 2011; Tennie et al. 2010): Personen, die zum Beispiel besonders viele Beiträge oder gut bewertete Beiträge einstellen, werden «ausgezeichnet» - sei es durch «Titel» wie «Top Contributor» oder durch Attribute, wie eine spezielle Farbe oder ein Distinktionssymbol, das dem Profilbild der Person hinzugefügt wird. Auch können besonders aktive Teilnehmende zusätzliche Funktionen erhalten, und werden auf diese Weise ebenfalls gegenüber den Anderen hervorgehoben.

Auf Community-Plattformen scheinen diese Mechanismen durchaus zu funktionieren und können - richtig angewendet - dazu beitragen, die Aktivität der Teilnehmenden zu fördern. Im Lernkontext sind hierzu noch keine klaren Befunde zu erkennen. Hier stellt sich das Problem, dass solche Mechanismen in der Regel erst ab einer gewissen Grösse einer Community greifen und vor allem dann von Bedeutung sind, wenn die Teilnehmenden einer Community sich nicht persönlich kennen, weitgehend anonym bleiben und häufig wechseln.

In vergleichsweise kleineren Lerngruppen mit stabilen Teilnehmenden, die sich noch dazu möglicherweise persönlich kennen (lernen), besteht die Gefahr, dass kontraproduktive Effekte entstehen, etwa dass die aktiven und «ausgezeichneten» Teilnehmenden immer mehr Beiträge liefern und die Nicht-Aktiven demotiviert werden. Auch kann ein solches «Ranking» von Lern-Kollegen und Kommilitonen/innen als befremdlich empfunden werden, zumal zu bedenken ist, dass in einer

5 Z.B. socialtext, grou.ps, cyn.in, drupal commons, elgg, socialwok 
überschaubaren Lerngruppe mit stabilen Teilnehmenden sich ohnehin recht schnell eine soziale Struktur bildet. Insofern ist die Frage der sozialen Akzeptanz eines «künstlich» aufgesetzten Reputationsmechanismus zu stellen

Eine Vielzahl von Lernaktivitäten lässt sich auf solchen Community-Plattformen organisieren und durchführen (vgl. Bosch 2009; Madge et al. 2009; Wang 2009). Gerade kollaboratives Arbeiten mit Artefakten aller Art lassen sich auf diesen Plattformen in der Regel gut realisieren. Gerade im Kontext informellen Lernens und nicht betreuter Varianten der Lernorganisation können solche Plattformen eine gute und effiziente Lösung sein, um Wissensgemeinschaften aufzubauen. An die Grenzen stösst dieser Ansatz immer dann, wenn spezifische und typische Funktionen eines LMS gewünscht oder notwendig werden: das Einstellen und Wiedergeben von Lernobjekten, die gesamte Abwicklung und Organisation von Prüfungs- und Testverfahren, das Einreichen und Bewerten von Lernleistungen, das Verwalten von Prüfungsergebnissen und eines Leistungs-Portfolios. Dies trifft insbesondere bei Bildungsangeboten zu, die von einer lehrenden Instanz organisiert und betreut werden. Hier wird man eher über eine «soziale Lernplattform» nachdenken.

\section{Soziale Lernplattformen}

Die dritte Variante zielt schliesslich darauf ab, eine (bestehende oder neue) Lernplattform mit dezidierten Funktionen für die Organisation des Lehr-Lernbetriebes um entsprechende Funktionen für soziale Kommunikation, Kollaboration und Community Building anzureichern. Tatsächlich bieten vorliegende Lernplattformen bereits heute eine Vielzahl entsprechender Funktionen bzw. integrieren diese zunehmend. Vertreter entsprechender Lösungen weisen in diesem Zusammenhang regelmässig darauf hin, dass ihre Lernplattformen viele oder alle diese Funktionen bereits beinhalten. Insofern ist im Folgenden genauer herauszuarbeiten, wie sich eine soziale Lernplattform von der «traditionellen» Nutzung von Lernplattformen unterscheidet. Dabei soll deutlich werden, dass sich ein traditionelles LMS von sozialen Lernplattformen weniger in den verfügbaren Funktionen unterscheiden, sondern vor allem in der Präsentationsschicht und dem Fokus, den die Plattform dem User präsentiert.

Traditionell wird in LMS die Idee sichtbar, Lernenden und Lehrenden Kursräume zu bieten, die klar definierte Grenzen, nämlich um den einzelnen Kursraum herum, aufweisen. Die Materialien werden üblicherweise in einen Kursraum bereitgestellt und die Kommunikation findet innerhalb der Grenzen des Kursraumes statt. Diese Struktur bietet für das Lernen einen geschützten Raum; sie ist einfach zu verstehen und kann von Lehrenden und Lernenden in der Nutzung sehr leicht nachvollzogen werden. Sie erinnert an das Klassenzimmer mit Tür, das von klar erkennbaren Wänden umgrenzt ist. 


\section{a) Aktivitäten im Fokus}

Bei sozialen Lernplattformen stehen dagegen die Lernenden und Lehrenden und ihre Aktivitäten im Vordergrund. Das System macht sichtbar, wer die Lernenden und Lehrenden sind, und zeigt ihre Aktivitäten. Im Hinblick auf Fragen des Datenschutzes mag man fragen, warum solche Informationen für Lernen überhaupt wesentlich sein sollten? Hier ist zu verweisen auf Theorien und Modelle des kooperativen Lernens, die die Potenziale des gemeinsamen Lernens und der sozialen Kommunikation für verschiedenartige Lernziele und Kompetenzen beschreiben und erklären. Gerade wenn es um Lehrziele geht, die über Fakten- und Regelwissen hinausgehen, erweisen sich entsprechende didaktische Methoden als besonders zielführend. Die Gruppenmitglieder bringen, auf dem Hintergrund ihrer Biografie, verschiedene Sichten ein. Ihre Erfahrungen und Kompetenzen helfen bei der Bearbeitung von Aufgaben und gemeinsamer Projekte. Hierbei können Fähigkeiten in der Diskussion und in der Teamarbeit entwickelt werden (vgl. Renkl und Mandl 1995).

Es zeigt sich, dass für diese Ansätze auch beim Online-Lernen bestimmte Erfolgsfaktoren gegeben sein müssen (vgl. Fischer et al. 2009). So benötigen die Lernenden regelmässig eine gewisse Zeit des Kennenlernens, über die sich wechselseitige Erwartungen über den Anderen entwickeln. Voraussetzung ist, dass sich grundlegendes Vertrauen bildet in den Anderen als Grundlage für eine Kontingenzreduktion in künftigen Interaktionen: Ich möchte wissen, mit wem ich es zu tun habe und ich möchte den Eindruck gewinnen, dass das Verhalten der Anderen meinen Normvorstellungen für Interaktionen entspricht (s. a. Zhan und Fang 2011). Gemeinsames Lernen im Austausch funktioniert nicht, wenn die Personen vollständig anonym bleiben würden und nicht sicher sind, dass die anderen Teilnehmenden identisch sind. Beim Online-Lernen ist es damit ebenfalls erforderlich, dass die Lernenden (und Lehrenden) sich zu einem gewissen Masse «kennen» müssen bzw. sollten. Dieses Kennenlernen kann face-to-face stattfinden, es kann aber auch vollständig im Internet stattfinden. Jeder Teilnehmer muss eine «Identität» aufweisen, und das bedeutet zunächst bereits, dass die Personen «identisch» bleiben müssen. Die Teilnehmenden erwarten, dass die Anderen über die Zeit die gleichen «ldentitäten» sind, da nur so die notwendige Kontingenzreduktion für gelingende soziale Interaktion vorliegt.

Es muss eine Motivation entstehen, warum die Lernenden Interesse an einem Austausch empfinden. Diese Motivation entsteht einerseits durch entsprechende Aufgabenstellungen, die die Lernenden auffordern, in einer Gruppe eine Aufgabe zu bearbeiten. Andererseits wird die Motivation auch beeinflusst davon, dass sich in der Interaktion Interesse entwickelt, mit bestimmten Personen zusammen zu arbeiten bzw. zusammen zu sein. Nach einer gewissen Zeit kann die Interaktion mit 
bestimmten Anderen selbst ein - wichtiger - Anreiz sein für die Lerntätigkeit bzw. für die Persistenz der Lerntätigkeit.

Dabei ist z. B. nicht wesentlich, dass die Lernenden mit ihrem bürgerlichen Namen bekannt sind, es reicht auch eine pseudonyme Identität aus, um entsprechende Gruppenprozesse in Gang zu bringen. Manche Personen werden im Internet unter einem Pseudonym berühmt, ohne dass ihr Name bekannt wird. Auch das Foto der Person ist nicht wesentlich, es sollte lediglich ein Bild (oder ein Avatar) vorliegen, das die Person einstellen kann. Auf diese Weise erzeugt der Lerner eine virtuelle Identität, die für die anderen Lernenden einen Wiedererkennungseffekt hat. Effekte der Eindrucksbildung, wie sie in der Sozialpsychologie untersucht werden, entstehen bei virtuellen Identitäten in ähnlicher Weise: Man entwickelt einen Eindruck über eine «Person», auch ohne wahre Daten über diese Person zu erfahren. Man entwickelt auch Sympathie oder Antipathie, beispielsweise wenn man Beiträge einer Person liest, oder beobachtet, ob sich eine «Person» pro- oder antisozial gegenüber anderen verhält (s. das Themenheft von Rummel und Krämer 2010).

\section{b) Permeabilität: Durchlässigkeit der Plattform zum Internet}

Die traditionelle Lernplattform definiert eine klare Grenze um den Kursraum. Dies hat, wie beschrieben, für Lehrende und Lernende deutliche Vorteile, weil es mental eine sparsame Konstruktion ermöglicht: Mir ist stets klar, ob ich mich innerhalb oder ausserhalb eines Kursraumes befinde. Mir ist stets klar, wo ein Dokument steht, das ich in den Kursraum geladen habe. Mir ist stets klar, was die Teilnehmer eines Kurses sehen und nicht. Der geschützte Lernraum bietet gerade aus pädagogischer Sicht zunächst wichtige Vorteile und die Möglichkeit, solche geschützten Lernräume einzurichten, kann geradezu als Voraussetzung für eine Lernplattform gelten: Lernen bedeutet immer auch, Fehler zu machen, und es sollte möglich sein, diese Fehler nicht dauerhaft im Internet sichtbar und für Alle zugänglich zu machen. Doch es gibt gute Gründe, über die Durchlässigkeit der Lernplattform nachzudenken. Dies kann in beide Richtungen erfolgen: aus dem Internet in die Lernplattform hinein, und aus der Lernplattform ins Internet.

Einbinden externer Ressourcen: Im Internet stehen zunehmend Materialien zur Verfügung, die für das Lernen nutzbar gemacht werden (können), zum Teil in den bereits erwähnten, in der Regel für den Nutzenden kostenfreien Diensten für das Einstellen von Dokumenten (wie YouTube, Slideshare etc.) oder in speziellen Datenbanken für Lernressourcen, wie z. B. Open Educational Resources (OER), oder iTunes University.

Dabei ist es vielfach weder sinnvoll oder möglich, solche Dokumente in die Lernplattform in einen Kurs hineinzukopieren. So ist es beispielsweise bereits relativ beschwerlich (und stellt juristische Fragen), ein Video von einer Plattform herunterzuladen, um es in eine andere Plattform - etwa mein LMS - hochzuladen, um 
es dort mit einem Player einstellen. Es ist wesentlich einfacher, das Video von der Ursprungsseite in meine Webseite einzubinden.

Werden Feeds von externen Quellen auf der Lernplattform eingebunden, wie z. B. bei Podcasts, so erhalten die Lernenden regelmässig die neuesten Beiträge der Feeds angezeigt, auch ohne dass diese auf der Plattform manuell aktualisiert werden müssten. Auch externe Informations- und Nachrichtendienste oder wissenschaftliche Zeitschriften lassen sich so auf der Lernplattform einbinden. Die Lernplattform bietet damit den Lernenden eine Umwelt mit sich ständig wechselnden und neuen Inputs, die zum Nachdenken und auch zum Reagieren auffordern.

Wichtig ist schliesslich, dass über diese Mechanismen auch Informationen, die Lernende ausserhalb der Plattform erzeugen, in die Lernplattform einbezogen werden und für andere Mitglieder der Lerngemeinschaft sichtbar werden: Foren- und (Micro-) Blogeinträge, Beiträge, die eine Person gelesen, gesehen, gehört oder bewertet hat - solche Spuren, die eine Person im Internet hinterlässt, können als «Strom von Aktivitäten» einer Person zusammengeführt werden und eben auch in der Lernplattform sichtbar gemacht werden.

Durchlässigkeit nach aussen: Die Lernplattform soll zunächst einen geschützten Raum bieten, in dem Lernende Fehler machen, etwas ausprobieren und Positionen entwickeln können, die nicht dauerhaft und für Alle sichtbar im Internet verbleiben. Dennoch kann überlegt werden, ob und an welchen Stellen es förderlich ist, Informationen aus der Lernplattform «nach aussen» verfügbar zu machen.

So ist zu bedenken, dass Lernende möglicherweise nicht ständig auf die Lernplattform gehen möchten, um sich über Neuigkeiten, Diskussionsbeiträge etc. zu informieren. Sie möchten möglicherweise über alternative Wege hierüber informiert werden bzw. über alternative Wege auf diese Informationen zugreifen. Eine einfache Variante besteht darin, den Lernenden differenziert über Neuigkeiten aus der Plattform per E-Mail zu informieren. Dabei ist es wichtig, dass die Person genau definieren kann, welche Arten von Informationen sie und wie oft erhält. Eine andere Variante besteht im Abonnieren der Neuigkeiten per Nachrichten-Feed. Hierbei ist zu prüfen, ob diese Feeds geschützt werden müssen, wenn sie Informationen nach aussen bringen, da ansonsten die Nachrichten öffentlich abgerufen werden können. Als weitere Variante wäre das Versenden von Nachrichten über Neuigkeiten als SMS an ein Mobiltelefon oder, etwa als private Nachricht, über einen Mikroblog-Service, wie z. B. Twitter.

Bei solchen Nachrichten besteht immer die grundsätzliche Frage: Soll das System die Nachricht selbst übermitteln oder soll nur mitgeteilt werden, dass eine Neuigkeit auf der Lernplattform bereitsteht? Teilnehmende erleben es als umständlich, manchmal sogar wie eine Schikane, wenn sie per Nachricht nur die Mitteilung erhalten, dass eine Nachricht im System vorliegt. Sie fragen, warum das System nicht direkt die Nachricht schickt? 
Bei manchen Informationen ist darüber hinaus zu beachten, dass die externen Kanäle die Anforderungen an Datenschutz zum Teil nicht zuverlässig gewährleisten. So wird man kaum Prüfungsergebnisse per E-Mail, SMS oder Twitter an eine Person senden, sondern höchstens die Information, dass eine Note im Lernstatus eingestellt worden ist.

\section{c) Abbildung von sozialen Beziehungen}

In der Lebenswelt sind Menschen in verschiedenen Rollen Mitglieder in mehreren sozialen Gruppen. In jeder Gruppe habe ich «Bekannte», die mir näher stehen, und mit denen ich mich enger austausche. Gruppen bilden hier ein spezifisches Konzept, um soziale Gemeinschaften und Beziehungen auszudrücken. Der Soziologe Tönnies grenzte Gemeinschaft ursprünglich von Gesellschaft ab:

Gemeinschaften bilden sich aufgrund einer familiären, räumlichen oder geistigen Nähe und schaffen eine Verbindung zwischen Menschen, die durch Zusammenleben und Anteilnahme am Anderen bestimmt ist (vgl. Tönnies 2005, 17 f). Die soziale Gruppe als soziale Kategorie kann nach Simmel zunächst durch den quantitativen Aspekt der Anzahl ihrer Mitglieder bestimmt werden (vgl. Simmel 1968, 32 ff.). Auch in anderen Eigenschaften unterscheiden sich soziale Gruppen, so dass die Zugehörigkeit eines Menschen zu unterschiedlichen sozialen Gruppen, in denen er sich im Laufe seines Lebens bewegt, seine Individualität entwickelt und ausdrückt (vgl. ebd., 312).

Aus der Theorie der sozialen Identität von Tajfel (1982) lässt sich ableiten, dass ein menschliches Bedürfnis existiert, Gruppen zuzugehören. Denn die eigene Selbsteinschätzung hängt zumindest in Teilen zusammen mit der Zugehörigkeit zu (mehreren) Gruppen sowie der Bewertung dieser Mitgliedschaften als mehr oder weniger erstrebenswert im Vergleich zu relevanten out-groups. Wird eine Gruppe, in der man Mitglied ist, als weniger attraktiv wahrgenommen als eine Gruppe, in der man nicht Mitglied ist, wird man z. B. versuchen, der anderen Gruppe beizutreten, die eigene Gruppe aufzuwerten oder andere Strategien der Konfliktbewältigung suchen. Damit wäre zum einen festzuhalten, dass ein grundlegendes Bedürfnis nach «Zugehörigkeit» zu sozialen Gruppen besteht, welche soziale Plattformen in Teilen befriedigen (s. a. Hölterhof und Kerres 2011).

Dazu muss die Plattform zumindest folgende Funktionen vorhalten:

- Erzeugen der Gruppengrenze: Die Plattform muss zunächst ermöglichen, dass Menschen in sozialen Gruppen organisiert werden. Sie muss Bereiche besitzen, in denen sich Lernende in Gruppen zusammenschliessen können und diesen Gruppen spezifische Funktionen anbieten. Der «Raum» einer Gruppe schafft damit für die Personen eine phänomenale Grenzziehung zwischen Mitgliedern und Nicht-Mitgliedern der Entität. Dabei können die Gruppen bestimmte 
Rollen und Beziehungstypen abbilden, die (technisch) mit unterschiedlichen Rechten und Aktivitäten in der Gruppe gekoppelt sein können. Die Identität der Gruppe kann zugleich mit weiteren Entitäten gekoppelt sein: So ist möglicherweise der Gruppenraum zugleich der Kursraum, in dem Lernmaterialien zur Verfügung stehen, oder eine Umgebung, in der an Projekten oder Fällen gearbeitet wird.

- Verbale Kommunikation: Die Menschen innerhalb der Gruppe müssen Wege finden, sich kommunikativ auszutauschen und zu verständigen. Synchrone Formate der Audio- und Videokonferenz nehmen dabei in Lernumgebungen an Bedeutung zu, auch asynchrone Formate, mit denen Audio- und Videobeiträge als Beiträge oder Kommentare in Foren eingestellt werden können. Dennoch dominiert weiterhin die textbasierte Kommunikation als primäre Form des Austausches in sozialen Gruppen, nicht zuletzt weil diese besonders schnell zu erfassen ist, und gut von mehreren Personen asynchron kommentier- und bewertbar ist. Die Beiträge und Kommentare werden dabei traditionell in Diskussionssträngen (threads) organisiert, in denen die wechselseitige Bezugnahme sichtbar wird. Eine andere Strukturierungsform textueller Kommunikation, die in Web-2.0-Anwendungen zunehmend dominiert, ist ihre Darstellung in zeitlicher Abfolge.

- Arbeiten mit Artefakten: Für alle Formen der Kooperation über das Internet wird es erforderlich, das die Gruppenumgebung Formen des Zusammenarbeitens ermöglicht oder unterstützt. Dabei kann sich die Zusammenarbeit auf ganz unterschiedliche Artefakte beziehen, auf Texte und alle anderen Arten von Dokumenten, auf Termine oder Programmcode. Sodann kann die Kooperation synchron und/ oder asynchron stattfinden.

In Lernplattformen werden Gruppen üblicherweise mit Kursräumen gleichgesetzt. Auf den ersten Blick bilden die Mitglieder einer Lehrveranstaltung eine soziale Gruppe und insofern erscheint es naheliegend, die soziale Kommunikation innerhalb einer solchen Lehrveranstaltung anzulegen. In dieser Weise werden z. B. Foren innerhalb eines Kursraumes eingerichtet und die Kommunikation der Lernenden innerhalb dieses Kursraumes angeregt und unterstützt. Damit bietet der Kursraum eine übersichtliche Umgebung für soziale Kommunikation, mit einer in der Regel definierten Zahl von Teilnehmenden, die ich zumeist überschauen kann und möglicherweise (aus Präsenzterminen) auch bereits persönlich kenne, und die ein gleiches Ziel verfolgen.

Eine solche Ausgestaltung sozialer Kommunikation in Foren innerhalb eines einzelnen Kursraumes erweist sich jedoch im Hinblick auf die Entwicklung einer Lerngemeinschaft als schwierig. Denn Community Building beinhaltet mehr als den fachlichen Austausch zwischen Kursteilnehmenden. Es geht auch um die persönli- 
che und emotionale Ebene, die Anteilnahme und Wertschätzung, die etwa durch einfache Formen der Bezugnahme («Danke für den Hinweis!») ausgedrückt wird. Für die Entwicklung einer Community sind auch Überraschungseffekte von Bedeutung, die die Plattform interessant machen: Reaktionen von Menschen, die man nicht kennt oder von denen man dies nicht erwartet hätte. In der eng umrissenen Umgebung des Kursraumes sind solche Effekte weniger wahrscheinlich. In der Forschung zu Hypertext wird von Serendipitäts-Effekten gesprochen (vgl. Schulmeister 1997), womit Zufallsfunde gemeint sind, die sich beim Browsen durch das Internet beiläufig ergeben und die für das (insbesondere: informelle) Lernen besonders fruchtbar sein können. Social Serendipity ist ein Effekt, der sich in sozialen Netzwerken ergibt, bei denen Personen zufällig auf andere Menschen, mit z. B. ähnlichen Interessen stossen, und sich mit diesen austauschen können. Dies erfordert eine hinreichend grosse Anzahl von Personen und auch Personen, die nicht unbedingt persönlich bekannt sind, weil sonst ein Überraschungseffekt fehlt.

\section{d) Optionen für Privatheit}

Wenn nun eine soziale Lernplattform grundsätzlich permeabel sein kann zu ihrer Umwelt, dann ist für verschiedene Arten von Lernaktivitäten zu entscheiden, wie diese für Andere innerhalb oder ausserhalb der Plattform sichtbar werden soll. Die Bedeutung von Privatheit und der Schutz von persönlichen Daten ist eine wichti-

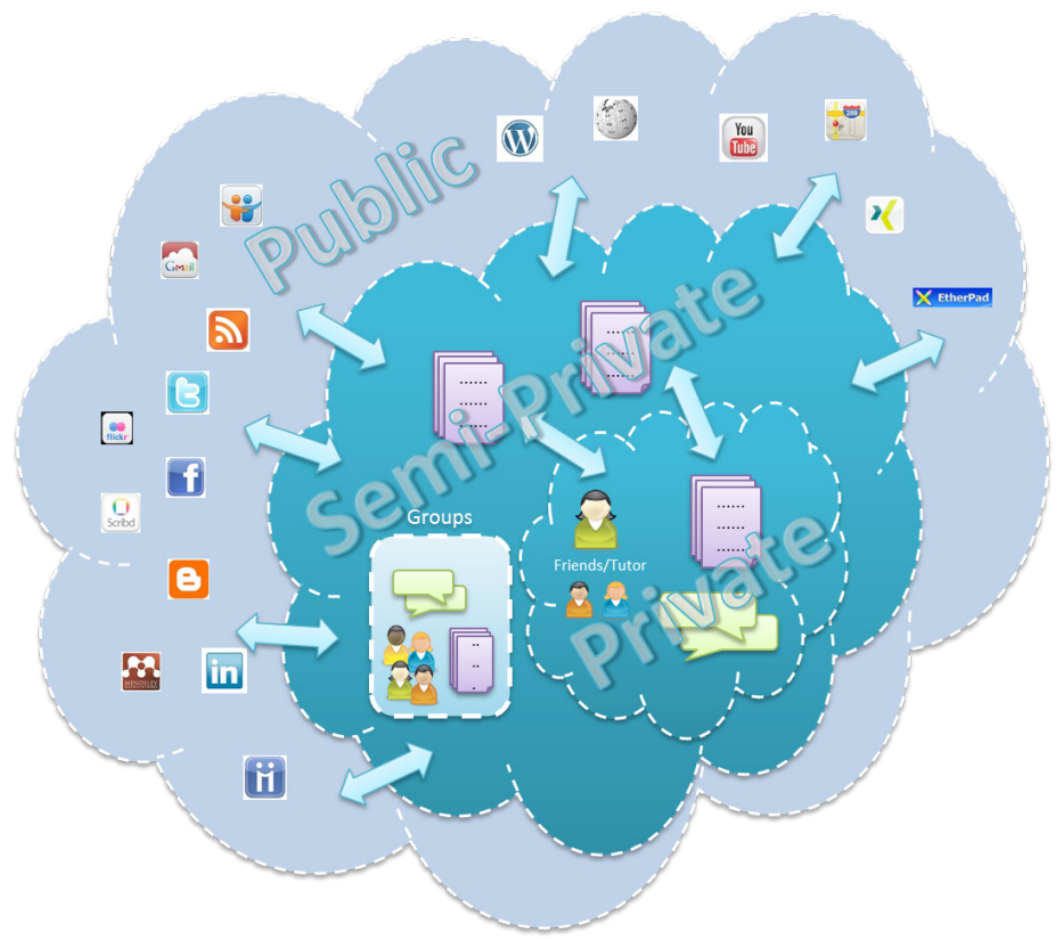

Abbildung 2: Lernen im privaten und öffentlichen Raum 
ge juristische und aktuelle gesellschaftspolitische Frage; sie betrifft aber zugleich auch didaktische Gestaltungsoptionen: Welche Varianten erscheinen aus mediendidaktischer Sicht für das Lernen und die Entwicklung des Lernenden und der Gruppe positiv?

Wir können dabei drei Grade der Öffnung unterscheiden: Private Lernaktivitäten sind nur dem Lernenden und möglicherweise der Lehrperson zugänglich. Teilweise private Lernaktivitäten sind beschränkt sichtbar für Mitglieder einer sozialen Gruppe, deren Mitglieder definiert und bekannt sind, etwa eine Schulklasse oder die Teilnehmenden eines Seminars. Die Jahrgangsstufe oder das ganze Matrikel der Studierenden, die sich in einem Semester in ein Studienfach eingeschrieben haben, könnten auch eine solche Gruppe bilden. In der Regel wird man diese Gruppe jedoch nicht für «semi-private Lernaktivitäten» heranziehen, wenn die Personen sich - wegen der Grösse der Gruppe - nicht mehr persönlich kennen, und ihre Kommunikation im Rahmen der unterrichtlichen Aktivitäten nicht wesentlich ist. Öffentliche Lernaktivitäten sind für «Alle» sichtbar (s. Abb. 2).

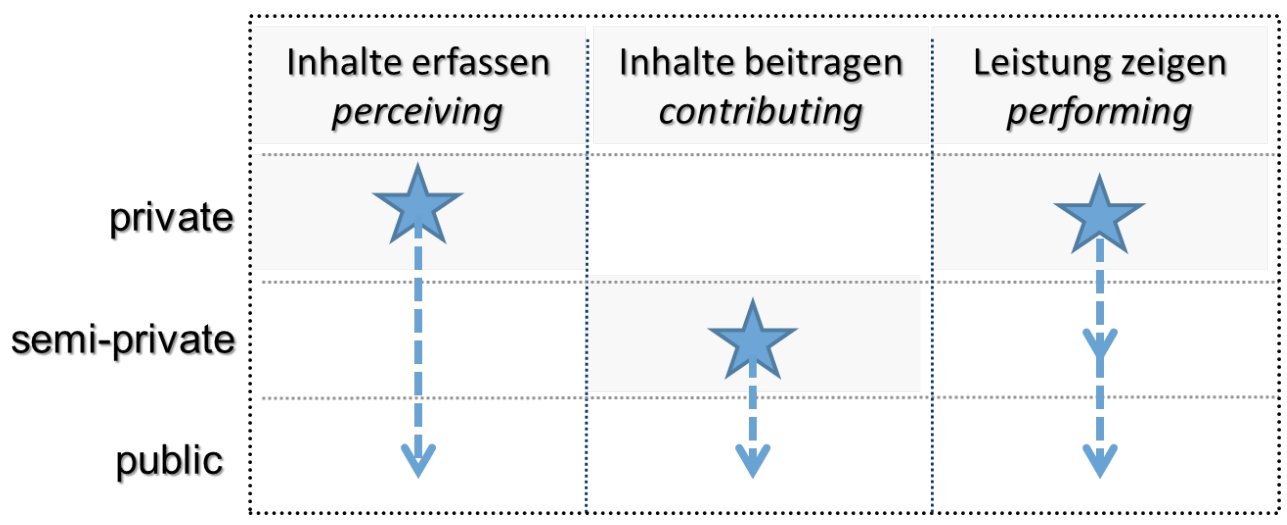

Abbildung 3: Optionen für Privatheit von Lernaktivitäten

\section{- Inhalte erfassen}

Das Lesen von Texten und Durcharbeiten von Materialien ist im traditionellen Unterrichtskontext eine Lernaktivität, die der Einzelne üblicherweise «privat» ausführt. Welches Buch ein Lerner aus der Schulbibliothek ausleiht und welche Seiten er daraus liest, wird auch der Lehrerin in der Regel nicht bekannt sein. Beim Lernen auf der Lernplattform können diese konkreten Lernaktivitäten auch «privat» verbleiben. Technisch ist es jedoch grundsätzlich nicht schwierig, diese Lernaktivitäten «sichtbar» für Andere zu machen. So können andere zum Beispiel sehen, wie oft ein Dokument heruntergeladen wurde, wie oft bestimmte Seiten aufgerufen wurden und auch wann und wo sich die Lernenden auf der Lernplattform bewegen und aufhalten. Bei sozialen Lernplattformen können diese Aktivitäten für Andere 
sichtbar gemacht werden oder sie können für Empfehlungssysteme ausgewertet werden, um etwa Hinweise zu geben: «Wer diese Seite gelesen hat, hat oft auch jene Seite aufgerufen...». E-Book-Reader besitzen zudem die Funktion, den Lesefortschritt öffentlich (z. B. über Twitter) zugänglich zu machen, so dass das Erfassen von Inhalten auch «öffentlich» ausgeführt werden kann.

Dabei ist zu beachten, dass für die "Observation» von Verhaltensdaten im Alltag deutliche Grenzen existieren, die durch rechtliche Rahmenbedingungen vorgegeben sind, und hier nicht im Einzelnen referiert werden können. In jedem Fall wird es der Entscheidung des einzelnen Lernenden zu überlassen sein, ob er oder sie Informationen über sich auf der Lernplattform einstellen bzw. für andere sichtbar machen will. Im Wesentlichen ist davon auszugehen, dass die Erfassung von Bewegungsprofilen, die einzelnen Personen zuordenbar sind, auch auf Lernplattformen grundsätzlich nicht zulässig ist. Wenn Zugriffe ausgewertet werden sollen, etwa für Empfehlungssysteme, so sind die zu verarbeitenden Informationen zunächst von einer zuordenbaren Person zu trennen und zu anonymisieren (insbesondere auch die zugehörige IP-Adresse zu entfernen). Dabei ist zu bedenken, dass diese Verfahren einige zusätzliche Verarbeitungsschritte erfordern, für die inhaltliche Auswertung und auch Empfehlungssysteme sind jedoch in der Regel ohnehin anonymisierte und aggregierte Informationen (z. B. Wie oft wurde die Seite angeklickt?) von Bedeutung bzw. hinreichend.

\section{- Inhalte beitragen}

Wenn es im nächsten Schritt darum geht, Inhalte beizutragen, etwa in einer Gruppen- oder Projektarbeit, dann sind diese Beiträge Anderen sichtbar, weil sie mit Anderen besprochen werden. In der Lernplattform beteiligen sich die Lernenden etwa in einem Forum, einem Chat oder in einer Online-Konferenz, sie stellen Beiträge etwa in einem Wiki, als Podcast oder in einer Fotogalerie auf der Lernplattform ein. Diese Beiträge sind den Teilnehmenden einer oder mehrerer Gruppen auf der Lernplattform sichtbar. Technisch ist es wiederum grundsätzlich möglich, diese auch öffentlich und weltweit zugänglich zu machen: Die erzeugten Artefakte können so von Anderen nachgehalten und weitergenutzt werden. Hier wird man im Einzelfall sehr genau überlegen (auch mit den Lernenden), inwiefern es didaktisch zielführend und wünschenswert ist, solche Beiträge tatsächlich öffentlich zu machen.

Um diese Beiträge auch ausserhalb der Lernplattform nutzen und verbreiten zu können, ist es vorteilhaft, diese über Feeds extern verfügbar zu machen. Auf diese Weise können Informationen unterschiedlicher Systeme auch zusammengeführt werden, in einem Feedreader, aber auch z. B. in einem E-Portfolio eines Studierenden. Dabei können auch mit Passwörtern geschützte Feeds realisiert werden, 
wenn diese Artefakte nicht «für Alle» öffentlich einsehbar werden sollen, sondern nur «von mir» in andere Systeme übertragen werden sollen.

\section{- Leistung zeigen}

Das Absolvieren von Prüfungen, wie Klausuren oder mündlichen Prüfungen, sind private Ereignisse, an dem in der Regel nur ein Lerner und sein Prüfer teilnehmen, möglicherweise noch ein Zweitgutachter oder Beisitzer. Werden Studienleistungen oder Prüfungsvorleistungen auf der Plattform eingestellt, können diese auch Anderen zugänglich gemacht werden. So wie ein Referat, dem die anderen Mitglieder, etwa einer Klasse, beiwohnen, kann zum Beispiel in einem Online-Kurs ein Blogbeitrag gefordert sein, der im Blogsystem der Plattform einzustellen und möglicherweise von Anderen zu kommentieren ist. Auch kann der Blogbeitrag in einem öffentlichen, externen Blog stehen, und die Person stellt den Link als Einreichung auf der Lernplattform ein. Dann wird die Leistung öffentlich sichtbar. Bei bestimmten Ergebnissen, gerade komplexerer Anforderungen, wie z. B. Projektarbeiten, kann es für die Lernenden durchaus motivierend und erwünscht sein, dass das Endergebnis öffentlich sichtbar wird, zumal hier in der Regel sehr viel Zeit investiert wird als in einem Beitrag im Rahmen einer Gruppendiskussion. Gleichwohl ist auch hier wichtig zu überlegen, welche Privatheitsoption pädagogisch sinnvoll ist.

Leistung zu zeigen ist folglich bislang in der Regel eine Aktivität, die der Lernende für sich vollzieht und der Lehrperson zugänglich ist. Durch soziale Lernplattformen können hier weitere Optionen realisiert werden, die vor allem dazu beitragen, dass auch alternative Studienleistungen leichter realisiert werden können. Das traditionelle und schlichte Einfordern eines «Essays» als Lernaufgabe, zu dem die Lehrperson dann eine Rückmeldung gibt, kann insbesondere durch Varianten des Peer-Assessments erweitert werden. Dabei werden die «Peers» (Mit-Lernenden) aufgefordert, zu Einreichungen Stellung zu nehmen und Rückmeldungen zu geben.

\section{Schluss}

Soziales Lernen wurde als eine Variante der Lernorganisation (und nicht als didaktische Methode) für das gemeinsame - auch unbetreute - Lernen beschrieben. Die traditionelle Anlage von Lernplattformen unterstützt soziales Lernen nur bedingt. Für institutionelle Bildungsangebote ist der Trend erkennbar, Lernplattformen stärker als «soziale Umgebungen» auszugestalten und den Austausch von Gruppen über Plattformen zu unterstützten. Der Beitrag hat aufgezeigt, welche didaktischen Gestaltungsfragen dabei entstehen. Dies betrifft u. a. die Frage der Sichtbarmachung von Aktivitäten der Lernenden sowie die Durchlässigkeit zum 
Internet und die damit zusammenhängenden Optionen für Privatheit. Dies betrifft auch die Frage, wie Bekanntschaften zu anderen abgebildet werden.

Communitys im Internet, in denen Menschen Wissen teilen und austauschen, finden teilweise grossen Zuspruch und schaffen neue Formen des Wissensaustausches, was auf ein Bedürfnis nach sozialem Lernen jenseits traditioneller Bildungsangebote hinweist. Menschen verbringen teilweise viel Zeit, um eigenes Wissen mit anderen zu teilen und ihnen bei ihren Fragen zu helfen. Manche Anbieter gehen auch konsequent den Weg, solche Plattformen für Communitys als Teil ihrer Bildungsarbeit zu betreiben und zu betreuen. ${ }^{6}$ Dabei darf nicht übersehen werden, dass in diesen Communitys auch bei mehreren tausenden Teilnehmenden oft nur ein kleiner Anteil sehr aktiv ist und die Community mit Leben füllt. Insofern ist es schwer, diese Phänomene übertragen zu wollen auf kleinere (Lern-) Gruppen im Kontext etwa von Lehrgängen. Gerade dort fällt es oft schwer, Lernende auf Lernplattformen zum Austausch und zu sozialer Kommunikation zu motivieren. Dies liegt zumeist nicht an mangelnden Kenntnissen und Fertigkeiten oder einer Ablehnung entsprechender Funktionen auf Seiten der Lernenden (für Studierende s. etwa die HIS-Studie von Kleimann et al. 2008). Die Lernenden nutzen oftmals andere Orte im Internet für ihren Austausch jenseits des von dem Bildungsanbieter organisierten und kontrollierten Lernraumes. Diese «Nebenwelt» kann als wichtige Funktion für die Lernenden, ihre Identitätsentwicklung und Sozialisation eingeschätzt werden. Es erscheint nur begrenzt machbar und erstrebenswert, sie einfach auf die Plattform des Bildungsanbieters übertragen zu wollen.

Auch wird man eine Reihe von Fällen finden, in denen solche sozial angelegten Lernumgebungen nicht zielführend sind, etwa wenn es um die eng vorstrukturierte Vermittlung von Fakten-, Konzept- und Regelwissen geht. Dennoch können, wie dargestellt, soziale Funktionen auch in solchen Fällen das Lernen unterstützen, ohne dass die Lernenden unmittelbar in Lern- und Gruppenaufgaben miteinander interagieren und kollaborieren.

6 z. B. evangelisch.de als Angebot der Evangelischen Kirche, das u. a. für den Wissens- und Erfahrungsaustausch Ehrenamtlicher dient 


\section{Literatur}

Baacke, Dieter. 1985. Handlungsorientierte Medienpädagogik. Hagen: Fernuniversität.

Bandura, Albert. 1971. Social Learning Theory. Morristown N.J.: General Learning Press.

Bandura, Albert. 1978. «Social Learning Theory of Aggression.» Journal of Communication 28 (3): 12-29.

Bosch, Tanja E. 2009. «Using online social networking for teaching and learning: Facebook use at the University of Cape Town.» Communicatio: South African Journal for Communication Theory and Research 35 (2): 185-200.

Brown, Stephen. 2010. «From VLEs to learning webs: the implications of Web 2.0 for learning and teaching.» Interactive Learning Environments 18 (1): 1-10.

Conole, Gráinne und Panagiota Alevizou. 2010. A Literature Review of the Use of Web 2.0 Tools in Higher Education: A report commissioned by the Higher Education. Milton Keynes, OK: Open University. http://www.heacademy.ac.uk/ assets/EvidenceNet/Conole_Alevizou_2010.pdf.

Downes, Stephen. 2010. "Learning Networks and Connective Knowledge.» ITForum 92 (2006): http://it.coe.uga.edu/itforum/paper92/paper92.html.

Fischer, Frank, Heinz Mandl und Albena Todorova. 2009. «Lehren und Lernen mit neuen Medien.» In Handbuch Bildungsforschung, hrsg. v. Rudolf Tippelt u. Bernhard Schmidt, 753-771. Wiesbaden: VS Verlag für Sozialwissenschaften. http://www.springerlink.com/content/v787xq10401g3g53/.

Grusec, Joan E. 1992. "Social Learning Theory and Developmental Psychology: The Legacies of Robert Sears and Albert Bandura.» Developmental Psychology 28 (5): 776-786.

Haake, Jörg, Gerhard Schwabe und Martin Wessner. 2011. CSCL-Kompendium 2.0: Lehr- und Handbuch zum computerunterstützten kooperativen Lernen. Oldenbourg: Wissenschaftsverlag.

Hölterhof, Tobias und Michael Kerres. 2011. «Modellierung sozialer Kommunikation als Communities in Social Software am Beispiel von Lernplattformen.» In Informatik schafft Communites: Proceedings der 41. Gl-Jahrestagung. Lecture Notes in Informatics (P-191): 433.

Kerres, Michael, Jörg Stratmann, Nadine Ojstersek und Annabell Preussler. 2009. «Digitale Lernwelten in der Hochschule.» In Digitale Lernwelten, hrsg. v. KaiUwe Hugger u. Markus Walber. Wiesbaden: VS Verlag für Sozialwissenschaften.

Kleimann, Bernd, Murat Özkilic und Marc Göcks. 2008. Studieren im Web 2.0. Studienbezogene Web- und E-Learning-Dienste. HISBUS-Kurzinformation (21). Hannover: HIS GmbH. https://hisbus.his.de/hisbus/docs/hisbus21.pdf.

Madge, Clare, Julia Meek, Jane Wellens und Tristram Hooley. 2009. «Facebook, social integration and informal learning at university: «It is more for socialising 
and talking to friends about work than for actually doing work ».» Learning, Media and Technology 34 (2): 141.

Nuissl, Ekkehard. 2010. "Ordnungsgrundsätze der Erwachsenenbildung in Deutschland.»In Handbuch Erwachsenenbildung/Weiterbildung, hrsg. v. Rudolf Tippelt und Aiga Hippel, 329-346. Wiesbaden: VS Verlag für Sozialwissenschaften. http://www.springerlink.com/content/jn59|56460uh4807/.

Oelkers, Jürgen. 1982. Soziales Lernen in der Schule. Königstein im Taunus: Scriptor Verlag.

Peters, Paul. 2011. Reputationsmanagement im Social Web: Risiken und Chancen von Social Media für Unternehmen, Reputation und Kommunikation. Köln: Social Media Verlag.

Petillon, Hanns. 1993. Soziales Lernen in der Grundschule: Anspruch und Wirklichkeit. Frankfurt am Main: Diesterweg.

Petschenka, Anke und Steffi Engert. 2011. «Einsatz von Lernplattformen in wissenschaftlichen Bibliotheken und Universitäten.» vdB-Mitteilungen 2011 (1).

Prenzel, Manfred, Heinz Mandl und Gabi Reinmann-Rothmeier. 1997. "Ziele und Aufgaben der Erwachsenenbildung». In Enzyklopädie der Psychologie: Psychologie der Erwachsenenbildung, hrsg. v. Franz E. Weinert und Heinz Mandl, 1-44. Göttingen: Hogrefe.

Renkl, Alexander und Heinz Mandl. 1995. "Kooperative Formen des Lehrens und Lernens in der Erwachsenenbildung.» Unterrichtswissenschaft 23 (4): 332-346.

Rummel, Nikol und Nicole Krämer. 2010. "Computer-Supported Instructional Communication: A Multidisciplinary Account of Relevant Factors.» Educational Psychology Review 22 (1): 1-7.

Salomon, Gavriel. 1993. Distributed Cognitions. Cambridge: Cambridge University Press

Salomon, Gavriel und David N. Perkins. 1998. «Individual and Social Aspects of Learning.» Review of Research in Education 23 (Januar): 1-24.

Schulmeister, Rolf. 1997. Grundlagen hypermedialer Lernsysteme. München: Oldenbourg.

Siemens, George. 2009. Handbook of Emerging Technologies for Learning. Winnipeg MB: University of Manitoba.

Tajfel, Henri. 1982. "Social Psychology of Intergroup Relations.» Annual Review of Psychology 33: 1-39.

Tennie, Claudio, Uta Frith und Chris D. Frith. 2010. «Reputation management in the age of the world-wide web.» Trends in Cognitive Sciences 14 (11): 482-488.

Tillmann, Klaus-Jürgen. 1976. Unterricht als soziales Erfahrungsfeld: soziales Lernen in der Institution Schule. Frankfurt am Main: Fischer Taschenbuch Verlag.

Tippelt, Rudolf. 2010. «Institutionenforschung in der Erwachsenenbildung/Weiterbildung.» In Handbuch Erwachsenenbildung/Weiterbildung, hrsg. v. Rudolf 
Tippelt und Aiga Hippel, 453-471. Wiesbaden: VS Verlag für Sozialwissenschaften. http://www.springerlink.com/content/m62405kq6128p7j5/.

Tönnies, Ferdinand. 2005. Gemeinschaft und Gesellschaft: Grundbegriffe der reinen Soziologie. Darmstadt: Wissenschaftliche Buchgesellschaft.

Wang, Minhong. 2009. «Integrating organizational, social, and individual perspectives in Web 2.0-based workplace e-learning.» Information Systems Frontiers 13 (2): 191-205.

Yang, Harrison. 2010. Collective Intelligence and E-Learning 2.0: Implications of web-based communities and networking. Hershey PA: Information Science Reference.

Zhan, Justin und Xing Fang. 2011. "Trust Maximization in Social Networks.» In Social Computing, Behavioral-Cultural Modeling and Prediction, hrsg. v. John Salerno, Shanchieh Jay Yang, Dana Nau und Sun-Ki Chai, 6589: 205-211. Berlin: Springer. http://www.springerlink.com/content/m7vg039365t27701/. 